\title{
Long Term Post Traumatic High Flow Carotid Cavernous Fistula with Patent Collateral Vessel: A Case Report
}

\author{
Luthfy Farhan ${ }^{1}$, Ridha Dharmajaya ${ }^{1}$ \\ ${ }^{1}$ Department of Neurosurgery,Universitas Sumatera Utara,Medan, Indonesia
}

\begin{abstract}
Introduction : A carotid-cavernous sinus fistula (CCF) is an abnormal communication between arteries and veins within the cavernous sinus. Carotid cavernous fistula $(\mathrm{CCF})$ is a very rare case it's difficult to diagnose. because most CCF patients rarely come for treatment.

Case Report : A 33-year-old male presented with history of protrusion of Left eye ball, and double vision for the last 2 years. visual disturbances were found in the right eye for 2 years, blurry vision is increasingly. Bruit was audible in orbital region on the left side. DSA showed that there was a fistula in the left sinus cavernous region, the arteries in the left area showed inadequate to direct the left hemisphere, but in the right arety showed that the right artery was adversely affected right and left brain.

Discussion : Traumatic CCFs are the most common type, accounting for up to $75 \%$ of all CCFs. 87 They have been reported to occur in $0.2 \%$ of patients with craniocerebral trauma and in up to $4 \%$ of patients who sustain a basilar skull fractur. ${ }^{2}$ The symptoms and signs of CCF always include eyelid swelling, proptosis, chemosis, and hyperaemia, dilated of vessel and the condition is commonly misdiagnosed as Graves'ophthal-mopathy or inflammatory conjunctivitis. ${ }^{3}$ Cerebral angiography is the gold standard for the definitive diagnosis, classification, and planning of endovascular intervention in CCFs. Angiographic results in this patient showed a fistula in the left cavernous sinus and inadequate supply of the left artery to the left hemisphere.

Conclusion : This case is very unique because the left brain gets blood supply from the right carotid system, with the left carotid artery system inadequate to direct the left hemisphere because of the carotid cavernous fistula on the left side
\end{abstract}

Keyword : Carotid Cavernous Sinus Fistula, Head Trauma, Vascular

\section{Introduction}

A carotid-cavernous sinus fistula (CCF) is an abnormal communication between arteries and veins within the cavernous sinus. CCFs may be classified into four types: direct fistulas (Barrow type A) and dural, or indirect, fistulas (Barrow types B, C, and D). Eighty percent incidens rate of CCFs were considered to be traumatic/type A with high flow velocities, and some standard textbooks still state that most cases of CCF occur after trauma[1]. However, the prevalence of nontraumatic CCF in these earlier publications is not constant in later reports, in the 1980s stated that the CCFs in $75 \%$ of the patients are spontaneous. The Clinical signs of CCFs is proptosis, Red eye with arterialization of the conjunctival and episcleral vessels, chemosis, strabismus due to ocular motor nerve dysfunction. Noninvasive imaging Computed tomography (CT), magnetic resonance (MR), CT scan of the orbit usually demonstrates proptosis of the affected globe, enlargement of the

*Corresponding author at: Department of Neurosurgery,Universitas Sumatera Utara,Medan, Indonesia 
extraocular muscles, dilatation and tortuosity of the superior ophthalmic vein (SOV), and enlargement of the ipsilateral cavernous sinus. Cerebral angiography is the gold standard for the definitive diagnosis, classification, and planning of endovascular intervention in CCFs[1][2].

\section{Case Report}

A 33-year-old male presented with history of protrusion of Left eye ball, and double vision for the last 2 years. Patient had suffered head injury due to road traffic accident 3 years back for which he was admitted in local hospital and was managed conservatively. visual disturbances were found in the right eye for 2 years, blurry vision is increasingly burdensome.On examination, there was protusion of left eye with dilated blood vessel in the sclera (figure 1). significant proptosis and Movements of eyeball were painful and restricted in all directions. Visual acuity was $6 / 6$ in the right eye and 6/60 in the left eye and fundus was papil edema in the left eye. Bruit was audible in orbital region on the left side.

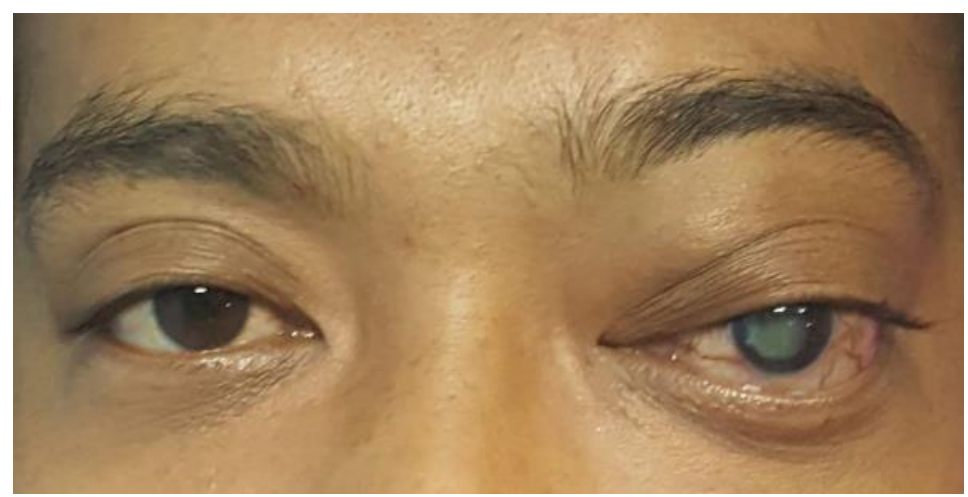

Figure 1. Proptosis on The left Eye with Positive Bruit

Computed tomography scan orbit showed proptosis of left eyeball with dilated tortuous superior ophthalmic vein. Vessel CT angiography showed right side direct high-flow CCF (Barrow type A) with dilated tortuous superior ophthalmic vein and marked corticovenous reflux.(figure 2)

Then we performed was Digital Subtraction Angiography (DSA), the results of our DSA showed that there was a fistula in the left sinus cavernous region, the arteries in the left area showed inadequate to direct the left hemisphere. In the right artery showed that the right artery was adversely affected right and left brain.(figure 2) 

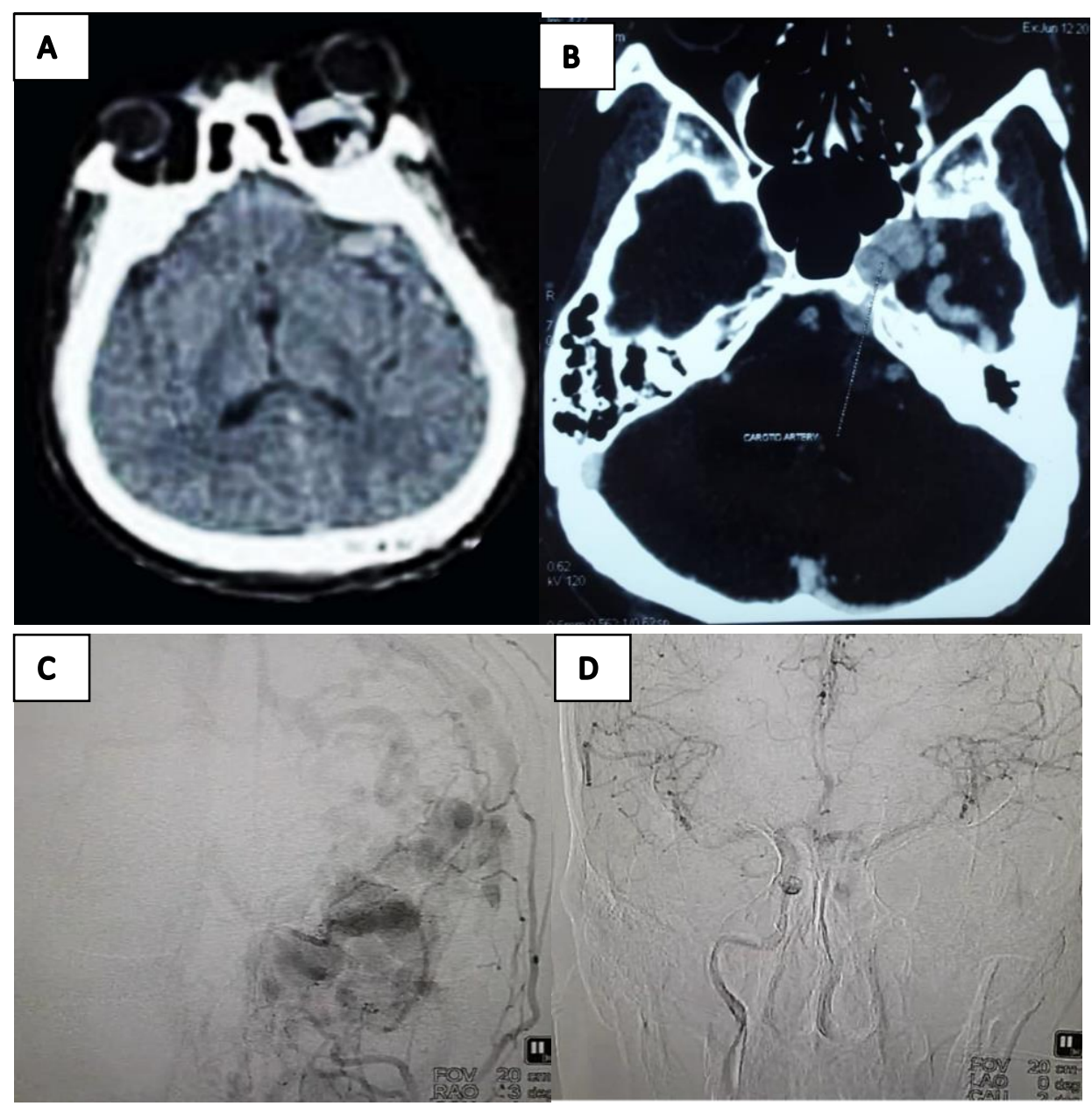

Figure 2. (A) Proptosis of left eyeball with dilated ophthalmic vein and marked cortico-venous reflux (B) Dilated tortuous superior tortuous superior ophthalmic vein (C) The arteries in the left area indequate (D) The right artery showed that the right artery was adversely affected right and left brain

\section{Discussion}

Traumatic CCFs are the most common type, accounting for up to 75\% of all CCFs.87 They have been reported to occur in $0.2 \%$ of patients with craniocerebral trauma and in up to $4 \%$ of patients who sustain a basilar skull fractur. [2] This patient has a history of trauma 3 years ago. The most frequent complaints involve the orbital region. The symptoms and signs of CCF always include eyelid swelling, proptosis, chemosis, and hyperaemia, dilated of vessel and the condition is commonly misdiagnosed as Graves'ophthal-mopathy or inflammatory conjunctivitis. [3] Physical diagnostic this patien show of proptosis, Dilation of blood vessels, disruption of the movement of the eyeball, according to the same symptoms of CCF. 
Noninvasive imaging [computed tomography (CT), magnetic resonance (MR), CT angiography, MR angiography, Doppler] often is used in the initial work-up of a possible CCF. CT scan of the orbit usually demonstrates proptosis of the affected globe, enlargement of the extraocular muscles, dilatation and tortuosity of the superior ophthalmic vein (SOV), and enlargement of the ipsilateral cavernous sinus. A noncontrast head CT scan also allows for careful examination of possible cranial injuries, such as bony fractures or intracranial hematomas. MR imaging findings in CCFs are similar to those seen on CT with the addition of orbital edema and abnormal flow voids in the affected cavernous sinus. In the setting of a high-flow fistula and retrograde cortical venous reflux, MR or CT studies may reveal dilatation of leptomeningeal and cortical veins. ${ }^{4}$ Computed tomography scan orbit showed proptosis of left eyeball with dilated tortuous superior ophthalmic vein. While CT angiography can be used as a first-line diagnostic tool in evaluating the presence of a CCF it has some limitations. Despite its ability to reliably delineate certain draining veins, CT angiography rarely depicts small feeding arteries in dural CCFs or the exact site of fistulous communication in direct CCFs. Moreover, this technique cannot provide information about the bloodflow characteristics within fistulas [4]

Vessel CT angiography showed right side direct high-flow with dilated tortuous superior ophthalmic vein and marked cortico-venous reflux. Cerebral angiography is the gold standard for the definitive diagnosis, classification, and planning of endovascular intervention in CCFs. The initial angiographic evaluation can be used to obtain the following information: size and location of the fistula, differentiation of direct from indirect lesions, presence of any associated cavernous carotid aneurysm, presence of complete or partial steal phenomena, assessment of the global cortical arterial circulation and collateral flow through the circle of Willis, identification of high-risk features (e.g., cortical venous drainage, pseudoaneurysm, cavernous sinus varix), venous drainage patterns, determination of therapeutic route, associated vascular injuries (e.g., traumatic pseudoaneurysm, arterial dissection), identification of any dangerous collateral pathways and evaluation of carotid bifurcation before compression therapy [5] Angiographic results in this patient showed a fistula in the left cavernous sinus and inadequate supply of the left artery to the left hemisphere.

\section{Conclusion}

A 33-year-old male presented with history of protrusion of Left eye ball, and double vision for the last 2 years. Patient had suffered head injury due to road traffic accident 3 years ago. Visual disturbances were found in the right eye for 2 years, blurry vision is increasingly 
burdensome.On examination, there was protusion of left eye with dilated blood vessel in the sclera, significant proptosis and Movements of eyeball were painful and restricted in all directions. Visual acuity was $6 / 6$ in the right eye and $6 / 60$ in the left eye and fundus was papil edema in the left eye. Bruit was audible in orbital region on the left side.

Computed tomography scan orbit showed proptosis of left eyeball with dilated tortuous superior ophthalmic vein., Vessel CT angiography showed right side direct highflow CCF (Barrow type A) with dilated tortuous superior ophthalmic vein and marked cortico-venous reflux.

Then we performed was Digital Subtraction Angiography (DSA), the results of our DSA showed that there was a fistula in the left sinus cavernous region, the arteries in the left area showed inadequate to direct the left hemisphere, in the right artery showed that the right artery was adversely affected right and left brain.

This case is very unique because the left brain gets blood supply from the right carotid system, with the left carotid artery system inadequate to direct the left hemisphere because of the carotid cavernous fistula on the left side. 


\section{References :}

[1]. Manuel Augusto Pereira Vilela, Carotid-cavernous fistula. 2017

[2] Linxin Zhu, Et al. (2018). Case Report : Post-traumatic right carotid-cavernous fistula resulting in symptoms in the contralateral eye: a case report and literature review

[3] Jason A. Ellis, M.D, Et Al. (2012). Carotid-cavernous fistulas Departments of INeurological Surgery and Radiology, Columbia University Medical Center, New York

[4] Bora Korkmazer, Burak Kocak, Et al. (2013). World Journal of Radiology : Endovascular treatment of carotid cavernous sinus fistula: A systematic review. ISSN $1949-8470$

[5] D. Rodriguez1, L. Aja Rodriguez, Et Al. (2014) European Society of radiology : Carotid-Cavernous Fistulas (CCF's): Imaging Features and Endovascular Treatment 